\title{
An analysis on Commercial and Cultural Functions of Fashion Show Spread
}

\author{
Rui Hou \\ Huanghe Science and Technology College \\ Zhengzhou, China
}

\begin{abstract}
Fashion show is a kind of communication activity, which is intended to disseminate fashion information, although with different forms and purposes, but in essence, it is the transmission of information. In this article, the author analyses the business and cultural transmission of fashion show from the perspective of communication sciences. It describes the communication model of fashion show: indicates that different types of performances need to choose different modes of transmission, such as commercial purposes or cultural purposes modes of transmission and so on. This article focuses on the business and social functions of fashion show. It presents an analysis of the results and function of dissemination with a few established theoretical models, and then it indicated the positive and negative social functions of fashion show.
\end{abstract}

Keywords-fashion show; business; culture communication; social function

\section{EXAMINING SEVERAL THEORIES AND FASHION SHOWS}

\section{A. "Theory of Silence Spiral" and Fashion Show}

1) Outline. "Silence Spiral" is a theory about the relationship between mass media and public opinion. This concept was first seen in an article of the German scholar Neumann in the "Journal of Communication," 1974. She found that: like other animals, humans have fear of isolation, based on this she proposed a theory of "silence spiral ".

The concept of "Silence Spiral " basically describes a phenomenon: when express their ideas and opinions, people will be actively involved and boldly express and diffuse of these ideas, if these ideas were agreed and widely welcomed; and for points which are of no or very few care (sometimes may cause oppose when expressed publicly), even if some people agree with these ideas but will remain silent. The silence of one party would cause momentum of the other party; over and over again, the voice of one party will be more and more powerful, and the other party more and more silent like the development of spiral.

Neumann proposed a powerful mass communication view based on the theory of "silence spiral ". This hypothesis consists of three elements: the formation of public opinion is the interaction result of mass communication, interpersonal communication and people's Cognitive Psychology on "Opinions environment", "public opinion may not be the 'majority' ones, but at least is the 'most' or 'superiority' opinions on the surface or in people's mind." Opinions prompted by the mass media have openness and communication, likely to be treated as "majority" or "advantage" opinions; pressure and sense of security brought by this situational awareness will cause spiral expansion in this interpersonal contacts "inferior views keep silence" and "advantage opinions speak loudly".

One of the important theoretical premises of "silence spiral" is the individual fear of social isolation and the convergence action of majority ideas caused by the fear. The theory of "Silence Spiral" emphasizes that the mass media have a powerful social effects and implications which provide a new perspective for the study of the effect of propagation science.

2) "Silence Spiral" in fashion show communication promotes fashion. Simmel considered the essence of fashion lied in only a portion of the population who led fashion, the whole group only follow suit. "Fashion is always what some people use; most of them are on the road to accept it." Fashion always generates change. When Fashion becomes widely popular, or for fashion only a few people pursue at first, without exception, affects all people, then it is no longer regarded as fashion, which can be understood that popular is the end of fashion. It can be said that the fashion show is the starting point of fashion, in order to obtain economic benefits by promoting fashion popular. Fashion show spreads out the latest fashion information and guide people to "follow suit."

Simmel also considered that "imitation" has become the most important driving force of fashion. Human behavior of Imitation stems from the fear of isolation. Fashion show, yet not a "majority" opinion, but is definitely "advantage" comment. When models wearing the latest fashion clothing appear in fashion shows, it takes for granted that their clothes are considered "advantage" of wearing. Models are better than ordinary people in physical condition with career elegant in pace and facial expressions; their body rhythms, temperament and demeanor are ordinary people elusive. This pulls away the model from the audience; the combining with clothing and model is more likely to be considered as "advantage" advice of fashion and then becomes the object of imitation. 
The function of trend releasing by fashion show determines that fashion show is bound to become a high class in fashion industry, and determines that the model's dress is bound to become the "advantage" opinion and then is sought as a fashion; as fashion is a kind of lower-level class imitation becoming popular, people fear that they would be "outdated" and throw out the old dress to follow fashion. Then fashion would be popular as a "majority opinion", it forces people with "disadvantage" comments in fashion to generate fear of social isolation and to follow fashion. At This time the fashion pioneer who has already produced new fashion guiding people to chase. This is a cycle, iterative process.

3) The function of "Agenda-setting" in fashion show. The function of "Agenda-setting" also exists in fashion show, existing with different ways and effects.

First of all, at the stage of spreading information in fashion show, fashion show disseminator controls information distribution and transmission; media of fashion show determine their audience, and arrange the agenda for audience. The most public attention information of fashion show must be published by the most influential communicators in fashion field. Audience is impelled by common brands and automatically takes well-known brands as a fashion icon. On the other hand, since the energy is limited in the medium audience and there are differences between the brand and the designer's strength, deviation might be caused on the audience's attention to fashion show, this is "celebrity effect" of function of agenda-setting; in addition, mass media of fashion show arrange information for audience, and release the information in the form of clothes with artificial ingredients, the strengthen of information makes them gain more public attention.

Secondly, the released information of fashion show reaches the ultimate goal of spreading by mass media. Fashion media organizations have a strong control power; audience can only choose from these media information passively. That is to say, the fashion media will get popular information according to the fashion show. The public appears uncertain when pursuing fashion, this is called the function of "agenda setting" in fashion show.

\section{THE FUNCTION OF TRANSMISSION AND COMMUNICATION EFFECT}

"The function of transmission is the ability to spread activities and its effect on people and the society." the function of Spread is not the same as the communication effect, if researchers see the effects of communication activities and energy release of media from social Angle and audience part, this belongs to "function" research; if the researchers appreciate the final results caused by communication activity and the reaction of the audience from the Angle of the communicators and the media itself this belongs to "effect" research.

Here we only analyze the social function related to the fashion show spread. Some important social function theories such as "agenda-setting" theory, "silence spiral theory", etc. have different performance in fashion show spread.

\section{EXAMINING The FASHION SHOW FunCtion BASED ON THE EXISTING THEORIES}

Fashion show proposed three functions for the commercial and cultural transmission from the Angle of sociology: endow social status, promote the practice of social norms, and negative function.

\section{A. "Social Status Awarded" Function of Fashion Show for Fashion}

"Social status awarded" function refers to: communication behavior can endow a certain social status for people, things and social activities, which makes social legitimation of the things and people, and sets up prestige, as well as receives a significant position, or on the contrary, it can make its reputation down.

There is no certain criterion for Fashion, whether it is fashion or backward, the result is relative. Fashion show played an important role in changing the face of fashion. How do communicators set "agenda-setting" for fashion, and make the agenda setting into reality, fashion show starts a make-up role. New fashion released in popular trend fashion show was identified as authoritative and at the cutting edge. The popular information appeared in fashion shows was given the significant position, if there is no fashion show, static display of works alone or image propaganda can't have obvious effect, then fashion is also impossible.

\section{B. "Compulsory Specification" Functions of Fashion Show \\ "compulsory Specification" function refers to the} communication in the mediation areas between the upper society and the vast majority of members, it can advertise some kind of social morality and social norms, and make them clear, finally achieve social recognition.

Spread of fashion show is the middle field between communicators and the public. Clothes deducted by models are considered to be beautiful dress. Models shape the public's aesthetic standards, and spread these standards through performance.

\section{Negative Social Functions of Fashion Show}

Practice of Spreading also shows that, as a kind mass media for fashion show, it also can produce negative function. For the sake of business profits, media make the information chaos, and cause people to lose bear ability in psychology, and social to lose sense of awareness. It is well known that: the dissemination of information will affect the audience, the influence may be positive or negative, and fashion show is no exception. For example, models are superior in figure; they guide the public to take thin for beauty which becomes the pursuit goal of people. In recent years, skeleton models appear in European fashion show, and they have become the darling of the runway. People pursue extreme weight loss to the point of malnutrition, they need to receive treatment. Then, negative thinking and 
measures have to be published...So, we must avoid the negative social functions brought by fashion show.

\section{CONCLUSION}

Any media, no matter what the specific content of spreading is, it brings certain information to human society, so it must play the role of guiding social change. Fashion show, as a kind of media is no exception. Analyzing fashion show in the principle of communication is a new attempt, the following are conclusions:

First, the author researches the medium characteristics of fashion show. Fashion show is a kind of communication activity, in essence, it is the information transmission no matter it is promotion fashion show, racing fashion show or entertainment fashion show, fashion show is an expanding and extension mean of information, so fashion show is also a kind of media, and has a special property different from traditional media: fashion show is a special organization communication activity rather than public communication activity.

Then, the author researches the model of fashion show. Commercial fashion show which takes guiding popular as the starting point, has a centralized top-down information transmission mode. Fashion show plays a great role in cultural transmission as the public "gatekeeper", in addition, fashion show also plays a role as "opinion leaders" - model, director, etc. It plays a positive and negative role actively and passively in the social and cultural transmission.

\section{REFERENCES}

[1] Yuan Yanyan, An Analysis of the Directing and Organization of Fashion Show [J], Art and Literature for the Masses, 2010, (13)

[2] Chen Lijuan, On the Fusion Beauty of Music and Fashion [J]. Journal of Northeast Dianli University (science and social edition), 2009,(3)

[3] Xiao Bin, An Interpretation of Creativity of Fashion Show from the Colorful Design of T Stage. Decoration, 2008,(4)

[4] Lu Hang, Magical Usage of Music at the Fashion Show[J]. Chinese businessman,2008,(14).

[5] Ma Xufei, Zhang Xiaoming, Zhou Ling, An Analysis of the Economic Effects of Fashion Show[J]. Modernization of business field, 2008, (21).

[6] Wu Zhiqin, On the Design and Organization of Fashion Show[J]. Silk Textile Technology Overseas, 2008,(1).

[7] Ma Xuefei, On Cultivation of Occupation Quality in College Students Majoring in Fashion Show [J]. Journal of Xingtai Polytechnic College,2007,(6).

[8] Liu Wenbo, Shi Jianping, On Fashion Show[J]. Silk Textile Technology Overseas, 2007, (4).

[9] Wu Zhiqin, On Art Teaching of Fashion Show and Shaping Students' Perfect Personality[J]. Textile Education, 2007, (3).

[10] Wu Yanhong, Fashion Style Is Basis of Forming Performance Style [J]. Hunan Baozhuang, 2006,(4).

[11] Guan Jie, An Appreciation of Fashion Show art [J]. Art Education, 2006, (10). 